\title{
Quantitative evaluation of olfactory dysfunction in hospitalized patients with Coronavirus [2] (COVID-19)
}

\author{
Georgios Tsivgoulis ${ }^{1,2}\left(\mathbb{D} \cdot\right.$ Paraskevi C. Fragkou $^{3} \cdot$ Alexander Delides $^{4} \cdot$ Emmanouil Karofylakis $^{3}$. \\ Dimitra Dimopoulou ${ }^{5} \cdot$ Petros P. Sfikakis ${ }^{6} \cdot$ Sotirios Tsiodras $^{3}$
}

Received: 28 April 2020 / Revised: 17 May 2020 / Accepted: 19 May 2020 / Published online: 25 May 2020

○) Springer-Verlag GmbH Germany, part of Springer Nature 2020

\section{Dear Editors,}

Severe acute respiratory syndrome coronavirus 2 (SARS$\mathrm{CoV}-2$ ) has spread in more than 200 countries, infecting almost 2,000,000 patients to date and causing the Coronavirus [2] (COVID-19) pandemic. There are recent reports indicating that COVID-19 patients commonly manifest olfactory and gustatory dysfunction even in the absence of rhinorrhea or nasal obstruction [1-4]. These preliminary observations are based on olfactory and gustatory questionnaires completed by patients and self-reported symptoms that are inherent to reporting bias and other limitations including reproducibility and validity. In addition, these reports included patients with minor COVID-19 symptoms and low-risk factor burden. In view of these considerations, we conducted a pilot case-control study using objective assessment of

Georgios Tsivgoulis and Paraskevi C. Fragkou equal contribution.

Georgios Tsivgoulis

tsivgoulisgiorg@yahoo.gr

1 Second Department of Neurology, School of Medicine, "Attikon" University Hospital, National and Kapodistrian University of Athens, Iras 39, Gerakas Attikis, 15344 Athens, Greece

2 Department of Neurology, University of Tennessee Health Science Center, Memphis, TN, USA

3 Fourth Department of Internal Medicine, Attikon University Hospital, National and Kapodistrian University of Athens, Athens, Greece

4 Second Department of Otolaryngology, School of Medicine, "Attikon" University Hospital, National and Kapodistrian University of Athens, Athens, Greece

5 Third Department of Pediatrics, "Attikon" University Hospital, National and Kapodistrian University of Athens, Athens, Greece

6 First Department of Propaedeutic Internal Medicine, National and Kapodistrian University of Athens Medical School, Athens, Greece olfactory function with a standardized microencapsulated odor identification test.

We prospectively included laboratory-confirmed COVID19 patients who were hospitalized because of pulmonary complications in the COVID-19 wards of a referral center in Athens ("Attikon" University Hospital) between March 19, 2020 and April 8, 2020. All cases included in the present study were diagnosed with COVID-19 using real-time reverse transcriptase-polymerase chain reaction (rRT-PCR) from upper respiratory samples. In detail, nasopharyngeal and/or oropharyngeal swabs were collected and transferred to the Clinical Microbiology laboratory, immersed in an appropriate virus transport medium (e.g. UTM Viral Transport, Copan Diagnostics Inc., Brescia, Italy). Flocked swabs made from synthetic material were preferred for sample collection to maximize viral recovery. Lower respiratory tract samples (e.g. bronchoalveolar lavage or aspirates, sputum, etc.) were also accepted. Automated purification of viral RNA from either viral transport medium or lower respiratory tract samples was performed using the QIAsymphony DSP virus/pathogen mini-kit on the QIAsymphony SP platform (QIAGEN, Hilden, Germany). A Real-Time, one-step Reverse Transcription-PCR, specific for ORF1ab gene of SARS-CoV-2 and for $\mathrm{N}$ gene of all, or other coronaviruses was finally performed on the Rotor-Gene Q MDx thermocycler (QIAGEN, Hilden, Germany), using the VIASURE SARS-CoV-2 Real-Time PCR Detection Kit (CerTest Biotec SL, Zaragoza, Spain).

We excluded patients (i) who reported olfactory disorder prior to the onset of COVID-19 respiratory symptoms, (ii) who reported a history of movement disorders, and (iii) patients who declined olfactory function testing. The very brief smell identification test (three-odorant test) chosen for this study was the Quick Smell Identification Test (Q-SIT; Sensonics, Inc.,Haddon Heights, NJ) and was selected on the basis of cost, convenience, format, standardization, and validation [5]. Q-SIT consists of individual $5 \times 5.5$ inch tear-out 
cards, each of which contains three microencapsulated odorant strips. Beside each strip is a multiple-choice question with five [5]. The Q-SIT score was computed by the sum of correct responses and ranges between 0 (anosmia) and 3 (normosmia). Q-SIT score values of 1 and 2 correspond to moderate/severe microsomia and mild microsomia [5]. We also evaluated nasal congestion using the SinoNasal Outcome Test 22 (SNOT 22) questionnaire that has been validated for use in Greek language [6].

Table 1 Baseline characteristics of cases $(n=22)$

\begin{tabular}{ll}
\hline Variable & \\
\hline Age, years (mean \pm SD) & $55 \pm 10$ \\
Male sex, $\%$ & $57 \%$ \\
Hypertension, $\%$ & $57 \%$ \\
Diabetes mellitus, $\%$ & $22 \%$ \\
Atrial fibrillation, $\%$ & $13 \%$ \\
Ischemic heart disease, $\%$ & $13 \%$ \\
Previous stroke, $\%$ & $0 \%$ \\
Smoking, $\%$ & $13 \%$ \\
Median number of risk factors (IQR) & $1(0-2)$ \\
Median numbers of days between symptom onset and & $12(9-15)$ \\
$\quad$ olfactory assessment (IQR) & \\
History of nose surgery, $\%$ & $9 \% *$ \\
History of chronic sinusitis, $\%$ & $5 \%$ \\
History of allergic rhinitis, $\%$ & $14 \%$ \\
\hline
\end{tabular}

$I Q R$ interquartile range
Controls included age- and sex-matched individuals who presented to Neurological Outpatient Clinic before COVID-19 outbreak (February 2020) in Greece with potential movement disorder symptoms and underwent evaluation of olfactory function using Q-SIT. The clinical diagnosis of Parkinson's Disease and other movement disorders were excluded in controls. All control patients were not hospitalized and were not affected by COVID-19. No control patient had influenza and common cold symptoms at the time of testing (January 2019-December 2019).

We included a total of 22 cases (mean age $55 \pm 10$ years, $57 \%$ men) and 22 age- and sex-matched controls. The baseline characteristics of the cases are summarized in Table 1. The median number of risk factors in cases was 1 [interquartile range (IQR) 0-2)] and the median number of elapsed days between hospital admission and olfactory testing was 12 (IQR 9-15). The median Q-SIT score was lower $(p=0.004)$ in cases (2, IQR: $1-2)$ than controls (3, IQR: 2-3; Fig. 1). The prevalence of normosmia $(p=0.006)$ was lower in cases (23\%) than controls (64\%). Gustatory dysfunction was very commonly or continuously self-reported in $23 \%$ of cases. Among the 16 cases with microsomia $(n=15)$ and anosmia $(n=1)$, nasal congestion was identified on SNOT 22 questionnaire in three patients. After excluding these three cases, the lower prevalence of normosmia persisted in cases $(26 \%)$ compared to controls $(64 \%, p=0.017)$ No patient with COVID-19 was intubated or died during their hospitalization.

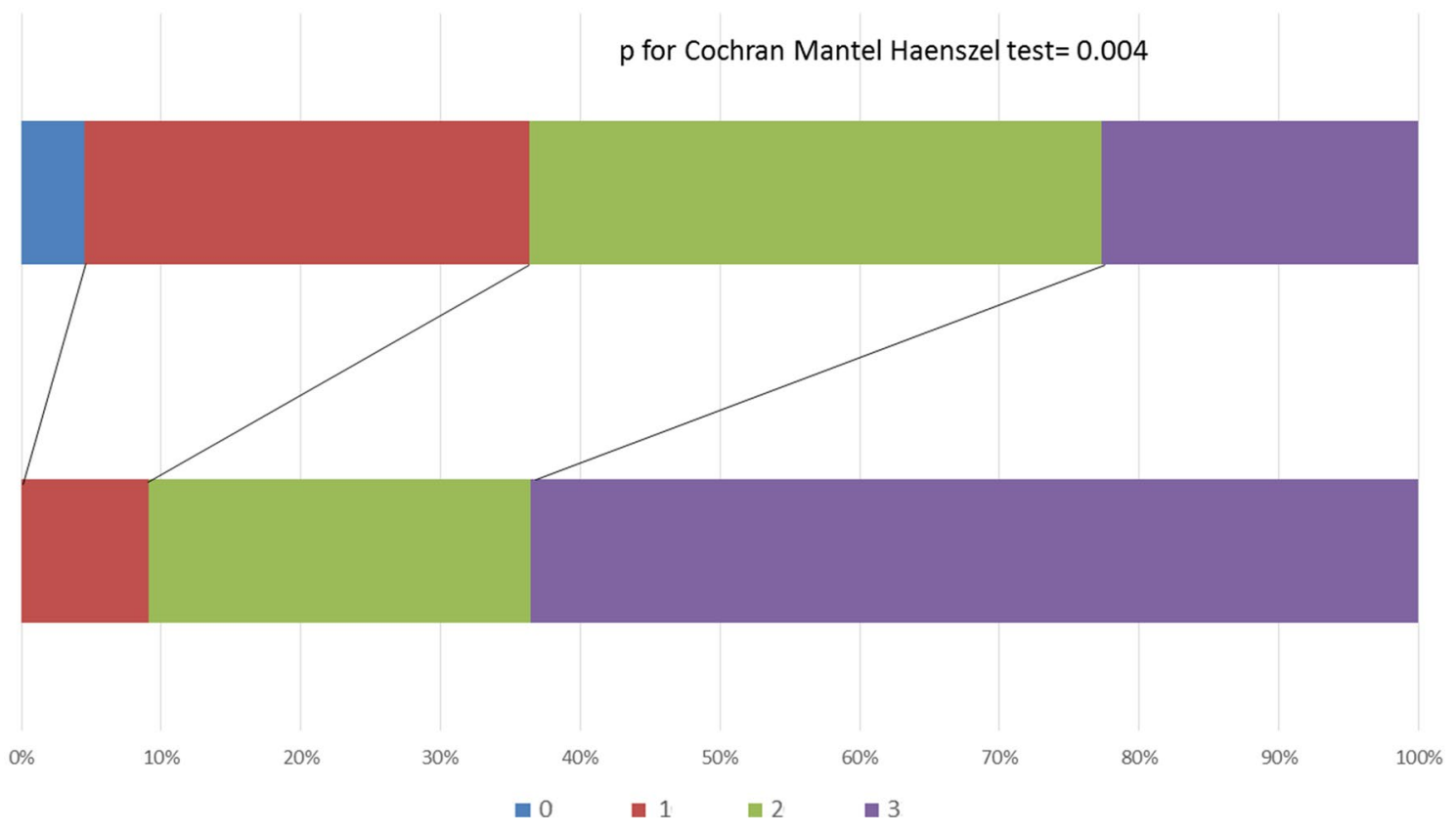

Fig. 1 Distribution of Q-SIT scores in cases and controls. Q-SIT scores ranged between 0 and 3 according to the number of correct answers. Statistical comparison between the two groups was per- formed using the Cochran-Mantel-Haenszel test. *Nasal septum surgery in $2016(n=1)$, rhinoplasty in $2014(n=1)$. IQR: interquartile range. 
The present study adds to the preliminary evidence provided by Moen et al. indicating that objectively assessed olfactory function may be impaired in COVID-19 hospitalized patients [7]. The findings of our pilot study highlight that moderate olfactory dysfunction was identified in approximately three-quarters of hospitalized COVID19 patients using an objective smelling identification test. Olfactory dysfunction was more prevalent in cases than controls even after controlling for nasal congestion symptoms. These findings lend support to the accruing literature underscoring olfactory dysfunction as a prevalent symptom of COVID-19 disease that should be systematically evaluated $[3,3]$. The potential movement of SARS-CoV-2 to the brain via the cribriform plate close to the olfactory bulb may represent a pathway enabling the virus to reach and affect the central nervous system that deserves further investigation [8].

Certain limitations of the present report should be highlighted including the small sample size, the lack of followup on the evolution of olfactory dysfunction, and the noninclusion of patients hospitalized in the Intensive Care Unit of our Institution. Another limitation is related to the lack of endoscopic/objective nasal examination to exclude the presence of unknown chronic rhinitis and nasal polyps. In brief, the present case-control study conducted in Greece coupled with the findings of a similar case-control study conducted in Iran [7] underscores that COVID-19 disease in hospitalized patients is commonly associated with microsomia that may be objectively assessed and quantified.

\section{Availability of data}

The raw data supporting the conclusions of this article will be made available by the authors upon reasonable request.

\begin{abstract}
Acknowledgements We would like to express our sincere gratitude to Dr. Ananstasia Antoniadou (ananto@med.uoa.gr), Dr. Christina Damoulari (damoulari_christina@outlook.com), Dr. Dimitra Kavvatha (dimitra.kavatha@gmail.com), Dr. Theodoros Marantos (marantos_ aek21@hotmail.com), Dr. Maria Paneta (mariathpaneta@gmail.com), Dr. Christos Psarrakis (chrispsarrakis@yahoo.com), Dr. Konstantinos Thomas (costas_thomas@yahoo.com), Dr. Georgios Tsioulos (geotsioulos89@gmail.com) and Dr. Nikolaos Tziolos (r-nikolaos.tziolos@ hotmail.com) for providing us the patients' clinical data and their daily input in the care of COVID-19 patients.
\end{abstract}

Author contributions GT, PF, AD, EK, DD collected the data. GT, PF, $\mathrm{AD}$ analyzed and interpreted the results. All authors wrote and revised the manuscript. All authors read and approved the final manuscript.
Funding None.

\section{Compliance with ethical standards}

Conflicts of interest Dr. Tsivgoulis: reports no conflicts of interest. Dr. Fragkou: reports no conflicts of interest. Dr. Delides: reports no conflicts of interest. Dr. Karofylakis: reports no conflicts of interest. Dr. Dimopoulou: reports no conflicts of interest. Dr. Sfikakis: reports no conflicts of interest. Dr. Tsiodras: reports no conflicts of interest.

Ethical standards This study has been approved by an appropriate ethics committee and has been performed in accordance with the ethical standards laid down in the 1964 Declaration of Helsinki and its later amendments.

Informed consent Written informed consent was obtained from the participants.

\section{References}

1. Mao L, Jin H, Wang M et al (2020) Neurologic manifestations of hospitalized patients with coronavirus disease 2019 in Wuhan China. JAMA Neurol. https://doi.org/10.1001/jamaneurol .2020.1127[Epub ahead of print] PubMed PMID: 32275288

2. https://www.medrxiv.org/content/10.1101/2020.03.23.20041 $889 \mathrm{v} 1$

3. Lechien JR, Chiesa-Estomba CM, De Siati DR et al (2020) Olfactory and gustatory dysfunctions as a clinical presentation of mild-to-moderate forms of the coronavirus disease (COVID-19): a multicenter European study. Eur Arch Otorhinolaryngol. https ://doi.org/10.1007/s00405-020-05965-1[Epub ahead of print] PubMed PMID: 32253535

4. Spinato G, Fabbris C, Polesel J et al (2020) Alterations in Smell or Taste in Mildly Symptomatic Outpatients With SARS-CoV-2 Infection. JAMA. https://doi.org/10.1001/jama.2020.6771[Epub ahead of print]

5. Jackman AH, Doty RL (2005) Utility of a three-item smell identification test in detecting olfactory dysfunction. Laryngoscope 115:2209-2212. https://doi.org/10.1097/01.mlg.0000183194 $.17484 . b b$

6. Lachanas VA, Tsea M, Tsiouvaka S, Hajiioannou JK, Skoulakis CE, Bizakis JG (2014) The sino-nasal outcome test (SNOT)22: validation for Greek patients. Eur Arch Otorhinolaryngol 271:2723-2728. https://doi.org/10.1007/s00405-014-2969-7

7. Moein ST, Hashemian SMR, Mansourafshar B, Khorram-Tousi A, Tabarsi P, Doty RL (2020) Smell dysfunction: a biomarker for COVID-19. Int Forum Allergy Rhinol. https://doi.org/10.1002/ alr.22587

8. Baig AM, Khaleeq A, Ali U, Syeda H (2020) Evidence of the COVID-19 virus targeting the CNS: tissue distribution, hostvirus interaction, and proposed neurotropic mechanisms. ACS ChemNeurosci 11:995-998. https://doi.org/10.1021/acschemneu ro.0c00122 